\title{
Article
}

\section{Magnetic Field Effect on a Radical Pair \\ Reaction as a Probe of Microviscosity}

Atom Hamasaki, Tomoaki Yago, and Masanobu Wakasa

J. Phys. Chem. B, 2008, 112 (45), 14185-14192• DOI: 10.1021/jp804862w • Publication Date (Web): 21 October 2008

Downloaded from http://pubs.acs.org on February 25, 2009

\section{More About This Article}

Additional resources and features associated with this article are available within the HTML version:

- $\quad$ Supporting Information

- Access to high resolution figures

- $\quad$ Links to articles and content related to this article

- Copyright permission to reproduce figures and/or text from this article

\section{View the Full Text HTML}




\title{
Magnetic Field Effect on a Radical Pair Reaction as a Probe of Microviscosity
}

\author{
Atom Hamasaki, ${ }^{\dagger}$ Tomoaki Yago, and Masanobu Wakasa* \\ Department of Chemistry, Graduate School of Science and Engineering, Saitama University, \\ 255 Shimo-okubu, Sakura-ku, Saitama-shi, Saitama 338-8570, Japan
}

Received: June 2, 2008; Revised Manuscript Received: July 26, 2008

\begin{abstract}
The magnetic field effects (MFEs), caused by the $\Delta g$ mechanism, on the photoinduced hydrogen abstraction reaction of benzopheneone with thiophenol were investigated in alcoholic solutions of varying viscosities $(\eta$ $=0.55$ to $59.2 \mathrm{cP}$ ) by a nanosecond laser flash photolysis technique. The escape yield of benzophenone ketyl radicals $(Y)$ gradually decreased with increasing magnetic field strength $(B)$ from 0 to $1.6 \mathrm{~T}$. The relative yield observed at $1.6 \mathrm{~T}, R(1.6 \mathrm{~T})=Y(1.6 \mathrm{~T}) / Y(0 \mathrm{~T})$, decreased with increasing $\eta$ in the range of $0.55 \mathrm{cP} \leq$ $\eta \leq 5 \mathrm{cP}$, and then increased with increasing $\eta$ in the range of $5 \mathrm{cP}<\eta \leq 55.3 \mathrm{cP}$. When $\eta$ was higher than $55.3 \mathrm{cP}$, the $R(1.6 \mathrm{~T})$ value became 1.0 , and MFEs were completely quenched. The observed $\eta$ dependence of the MFEs was analyzed by the stochastic Liouville equation (SLE), in which the effects of spin-orbit coupling by a heavy atom such as sulfur were taken into account. The observed MFEs were reproduced fairly well by the SLE analysis. The diffusion coefficients of the radicals obtained by the SLE were about three times smaller than those expected from the macroscopic solvent viscosities. One can probe the microviscosity in the vicinity of the radical pairs by observing MFEs on the present photochemical reaction system.
\end{abstract}

\section{Introduction}

Magnetic field effects (MFEs) on photochemical reactions through radical pairs (RPs) and biradicals have received considerable attention during the past three decades. ${ }^{1-3}$ Magnetic fields interact with the electron spins of RPs, and thus the spin conversion in the RPs is influenced by the fields. The lifetime of the RPs and the yield of the escaped radicals consequently show appreciable MFEs. In solution, the MFEs on the reactions of RPs have been interpreted in terms of the radical pair and triplet mechanisms (RPM and TM, respectively). According to the RPM, MFEs are observed during sequential steps as follows: (1) formation of close RPs through photochemical reactions with singlet (S) or triplet $\left(\mathrm{T}_{n}, n=0, \pm 1\right)$ spin multiplicity, (2) spin conversion between $\mathrm{S}$ and $\mathrm{T}_{n}$ states in separated RPs, and (3) spin state selective recombination of the close RPs competing with the escape of radicals from the pairs. ${ }^{2}$ These steps are promoted by the diffusion of the radicals by which the RPs are separated and re-encountered. Radical diffusion, therefore, has a pronounced effect on the magnitude of the observed MFEs. Large MFEs have been observed in confined and inhomogeneous systems such as micellar solutions, ${ }^{2,3}$ highly viscous solutions, ${ }^{4,5}$ nanotubes, ${ }^{6}$ and very recently ionic liquids. ${ }^{7}$

Molecular and radical diffusions are important processes that control chemical reactions in solutions. ${ }^{8}$ Radical diffusion motion has been studied with the several techniques, including transient grating (TG), ${ }^{9}$ photochemical space intermittency, ${ }^{10,11}$ and electron paramagnetic resonance (EPR). ${ }^{12,13}$ These techniques provide valuable information on radical diffusion in homogeneous solutions. Using the TG technique, for example, Terazima et al. found that photochemically generated radicals diffuse more slowly than their parent molecules. ${ }^{9}$ In such techniques, the observed radical diffusion is averaged over a

\footnotetext{
* To whom correspondence should be addressed. E-mail mwakasa@ chem.saitama-u.ac.jp.

${ }^{\dagger}$ Present address: Department of Chemistry, Faculty of Science, Shinshu University, 3-1-1 Asahi, Matsumoto, Nagano 390-8621, Japan.
}

certain time period, which is typically longer than microseconds. Thus, the obtained diffusion coefficient of radicals is associated with macroscopic solvent viscosity, and it is difficult to probe the fast diffusion of radicals in the localized structures of confined and inhomogeneous systems.

Since the interactions of electron spins in RPs are limited to within a few nanometers, the absolute magnitude of the MFEs is affected by the microviscosity in the vicinity of the RPs rather than by the macroscopic viscosity. Therefore, the microviscosity in confined and inhomogeneous systems may be probed by observing MFEs. Although studies of MFEs on RPs demonstrate great promise for this application, only a few systematic studies on the viscosity $(\eta)$ dependence of MFEs exist, even for homogeneous solvents. ${ }^{4,14,15}$ Wolff et al. reported a $\eta$ dependence of MFEs in electron-transfer reaction systems involving Ru-trisbipyridine, although the mechanism explaining the dependence was different from the present study..$^{15}$ The obtained results have been interpreted in terms of the change of the dielectric solvent relaxation or the donor-acceptor distance dependence of the charge recombination rate. Regarding neutral RPs, Wakasa et al. reported the $\eta$ dependence of MFEs on the hydrogen abstraction reaction of benzophenone (BP) with thiophenol $(\mathrm{PhSH})$ in nonviscous homogeneous solutions. ${ }^{14}$ The absolute magnitude of the MFEs increased with increasing $\eta$. The observed MFEs were explained well by the simple diffusion model developed by Freed and Pedersen. ${ }^{16,17}$ In a similar photochemical reaction of 4-methoxybenzophenone (MBP) with 4-chlorothiophenol $(\mathrm{ClPhSH})$ in highly viscous solvents, $\mathrm{Ki}$ tahama et al. reported that the absolute magnitude of MFEs decreased with increasing $\eta$, although the diffusion model predicted increases of MFEs with increasing $\eta$ together with a saturation of MFEs in highly viscous solvents. ${ }^{4}$ Therefore, the origin of the observed MFE quenching in highly viscous solvent has not been clarified yet, and the $\eta$ dependence of MFEs should be studied in greater detail. 
TABLE 1: Viscosities $(\eta)$ of Pure Solvents

\begin{tabular}{cc}
\hline solvent & $\eta / \mathrm{cP}^{a}$ \\
\hline methanol & 0.55 \\
ethanol & 1.08 \\
2-propanol & 2.04 \\
2-methyl-1-propanol & 3.33 \\
&
\end{tabular}

${ }^{a}$ Reference 18.

TABLE 2: Viscosities $(\eta)$ of Mixed Solvents of 2-Methyl-1-propanol $(i$-BuOH) and Cyclohexanol $(c$-HexOH)

\begin{tabular}{cc}
\hline mixed ratio $i$-BuOH:c-HexOH $(v / v)$ & $\eta / \mathrm{cP}^{a}$ \\
\hline $2 / 1$ & 6.62 \\
$1 / 1$ & 9.65 \\
$1 / 2$ & 16.9 \\
$1 / 5$ & 30.2 \\
$1 / 9$ & 37.3 \\
$1 / 50$ & 55.3 \\
$1 / 100$ & 59.2
\end{tabular}

${ }^{a}$ Obtained from the kinetic viscosities and densities at $296 \mathrm{~K}$.

Recently, we investigated MFEs on the photoinduced hydrogen abstraction reaction of $\mathrm{BP}$ with $\mathrm{PhSH}$ with a wide range of $\eta$, from 0.55 to $59.2 \mathrm{cP}$, using several alcohols and their mixtures. In this study, the $\eta$ dependence of MFEs caused by the $\Delta g$ mechanism $(\Delta g \mathrm{M})$ was investigated, and we report the detailed analysis of MFEs using the stochastic Liouville equation (SLE). The MFEs observed were well-reproduced by the SLE simulation. We demonstrate that the microviscosity in confined and inhomogeneous systems can be probed by observing MFEs with the present photochemical reaction.

\section{Experimental Section}

Materials. Benzophenone (BP, Cica) was recrystallized twice from methanol. Thiophenol $(\mathrm{PhSH}$, Cica) was purified by vacuum distillation. Methanol (MeOH, Cica), ethanol (EtOH, Cica), 2-propanol (2-PrOH, Cica), 2-methyl-1-propanol ( $i$ $\mathrm{BuOH}, \mathrm{Cica})$, and cyclohexanol $(c-\mathrm{HexOH}$, Cica) were used as received. Mixtures of several ratios of $i-\mathrm{BuOH}$ and $c-\mathrm{HexOH}$ were used to create highly viscous solvents. The concentrations of BP and PhSH were $2.0 \times 10^{-2}$ and $1.2 \times 10^{-1} \mathrm{~mol} \mathrm{dm}^{-3}$, respectively.

The viscosity and density of each solvent were measured by a Yamauchi VM-10A-L viscometer and an Anton Paar DMA 5000 density meter. The viscosities of the solvents employed in the present study are listed in Tables $1^{18}$ and 2 . All solvent ratios reported herein are $v / v$ ratios.

Nanosecond Laser Flash Photolysis. Laser flash photolysis experiments were carried out with an apparatus that was essentially the same as the apparatus described elsewhere. ${ }^{14 a, 19}$ The third harmonic $(355 \mathrm{~nm})$ of a Nd:YAG laser (Quanta-Ray INDY) with a pulse width of $7 \mathrm{~ns}$ was used as an exciting light source. The probe light from a Xe short arc lamp (Perkin-Elmer Optoelectronics Lx-300 or Ushio UXL-500D) with a custombuilt pulse current generator was divided into two beams, creating a double-beam probe system. One beam was guided to a quartz sample cell by a quartz optical fiber (3 M FT1.0UMT) and passed through the cell. The other was detected directly. Both beams were guided by optical fibers to photomultipliers (Hamamatsu R636-10) through a monochromator (ORIEL MS257 and SHIMADZU SPG-120S, respectively). This double-beam probe system was constructed to accurately observe transient absorption by maintaining a flat baseline signal. However, in the present study, the baseline contained somewhat large noise contributions, which were generated from Qswitching of the laser and the pulsed trigger of the Xe lamp. Signal voltage from the photomultiplier was terminated by a $50 \Omega$ resistor and was recorded by a LeCroy Wave Pro 960 digitizing oscilloscope $(2 \mathrm{GHz})$. A personal computer was used to control the apparatus and record data. Magnetic fields of up to $1.6 \mathrm{~T}$ were provided by a Tokin SEE-10W electromagnet. The quartz cell was placed at the center of the electromagnet. Argon-bubbled solutions were circulated through the quartz cell at a rate of $20 \mathrm{~mL} / \mathrm{min}$. Laser flash photolysis measurements were carried out at $296 \mathrm{~K}$.

\section{Results and Discussion}

Reaction Scheme. The following reactions occur by the photoexcitation of $\mathrm{BP}$ in the presence of $\mathrm{PhSH}:{ }^{14 \mathrm{a}}$

$$
\begin{gathered}
\mathrm{BP}+h v(355 \mathrm{~nm}) \rightarrow{ }^{1} \mathrm{BP} * \rightarrow{ }^{3} \mathrm{BP}^{*} \\
{ }^{3} \mathrm{BP} *+\mathrm{PhSH} \rightarrow{ }^{3}(\mathrm{BPH} \bullet \bullet \mathrm{SPh}) \\
{ }^{3}(\mathrm{BPH} \bullet \bullet \mathrm{SPh}) \stackrel{{ }^{B}}{\leftrightarrow}(\mathrm{BPH} \bullet \bullet \mathrm{SPh})
\end{gathered}
$$

$1(\mathrm{BPH} \bullet \bullet \mathrm{SPh}) \stackrel{k_{\text {rec }}}{\longrightarrow}$ recombination products

$$
3,1(\mathrm{BPH} \bullet \bullet \mathrm{SPh}) \stackrel{k_{e s c}}{\longrightarrow} \text { escaped radicals }
$$

Here, ${ }^{1} \mathrm{BP} *$ and ${ }^{3} \mathrm{BP} *$ represent the singlet and triplet excited states of $\mathrm{BP}$, respectively. $\mathrm{BPH} \bullet$ and $\bullet \mathrm{SPh}$ represent the benzophenone ketyl and phenylthiyl radicals, respectively. ${ }^{1}(\mathrm{BPH} \bullet \bullet \mathrm{SPh})$ and ${ }^{3}(\mathrm{BPH} \bullet \bullet \mathrm{SPh})$ denote singlet and triplet RPs, respectively, that are composed of $\mathrm{BPH} \bullet$ and $\bullet \mathrm{SPh}$. Upon laser excitation of $\mathrm{BP},{ }^{3} \mathrm{BP} *$ is immediately generated via the fast intersystem crossing of ${ }^{1} \mathrm{BP} *$ (eq 1). The triplet RP is formed by a hydrogen abstraction reaction of ${ }^{3} \mathrm{BP} *$ with $\mathrm{PhSH}$ (eq 2). The MFEs caused by the $\Delta g \mathrm{M}$ are, more specifically, caused by the difference between the isotropic $g$ factors $(\Delta g)$ of two radicals in a pair. Here, the spin mixing rate $\left(k_{\Delta g \mathrm{M}}\right)$ between the $\mathrm{S}$ and $\mathrm{T}_{0}$ states is simply proportional to $\Delta g$ and the magnetic field strength $(B)$.

$$
k_{\Delta g \mathrm{M}}=\frac{1}{2} \hbar^{-1} \Delta g \mu_{\mathrm{B}} B
$$

Thus, the magnetic field accelerates the spin conversion between singlet and triplet RPs by the $\triangle g \mathrm{M}$ (eq 3). The recombination of singlet RPs proceeds with a rate constant $k_{\text {rec }}$ (eq 4 ), whereas the radicals escape from the singlet and triplet RPs with a rate constant $k_{\text {esc }}$ (eq 5).

Measurements of Transient Absorption. The laser flash photolysis of BP $\left(2.0 \times 10^{-2} \mathrm{~mol} \mathrm{dm}^{-3}\right)$ with $\mathrm{PhSH}(1.2 \times$ $10^{-1} \mathrm{~mol} \mathrm{dm}^{-3}$ ) in each alcohol solution was performed at 296 $\mathrm{K}$. Time profiles of the transient absorption $(A(t))$ were measured in the wavelength range of 360-700 $\mathrm{nm}$. Transient absorption spectra observed in a $1 / 9$ mixture of $i-\mathrm{BuOH}$ and $c-\mathrm{HexOH}(\eta$ $=37.3 \mathrm{cP}$ ) at delay times of $10,30,80$, and $200 \mathrm{~ns}$ after laser excitation are shown in Figure 1. A transient absorption peak due to the triplet-triplet $(\mathrm{T}-\mathrm{T})$ absorption of ${ }^{3} \mathrm{BP} *$ was observed at $525 \mathrm{~nm} .{ }^{20}$ This peak decayed quickly, with a rate constant of $6.0 \times 10^{7} \mathrm{~s}^{-1}\left(\tau_{\mathrm{T}-\mathrm{T}}=17 \mathrm{~ns}\right)$, and the decay was accelerated with increasing $\mathrm{PhSH}$ concentration. Figure 2 shows the $\mathrm{PhSH}$ concentration dependence of the ${ }^{3} \mathrm{BP} *$ decay rate constant $(k)$ observed at $525 \mathrm{~nm}$ in the $1 / 9$ mixture $(\eta=37.3$ cP) for PhSH concentrations of $0.03-0.6 \mathrm{~mol} \mathrm{dm}^{-3}$. A good 


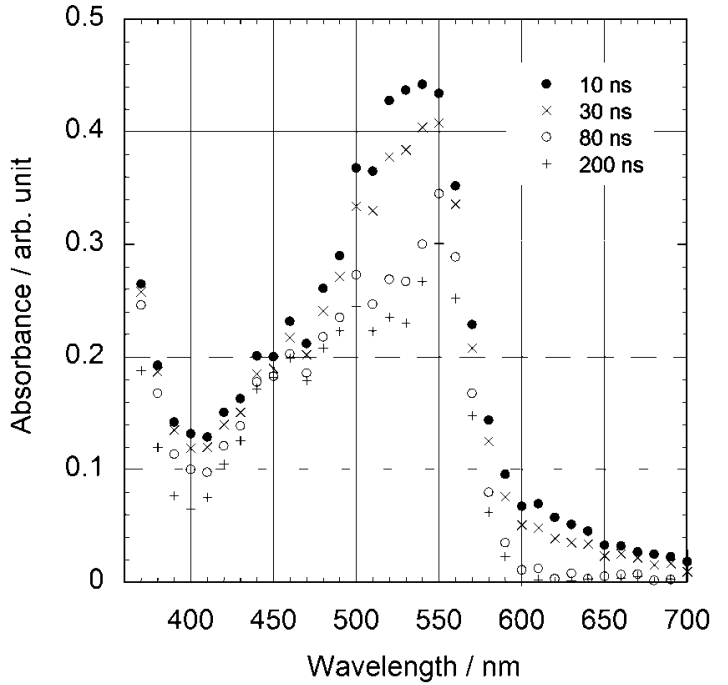

Figure 1. Transient absorption spectra observed at delay times of 10 , 30,80 , and $200 \mathrm{~ns}$ after laser excitation of a 1/9 mixture of $i$-BuOH and $c$-HexOH $(\eta=37.3 \mathrm{cP})$ containing $\mathrm{BP}\left(2.0 \times 10^{-2} \mathrm{~mol} \mathrm{dm}^{-3}\right)$ and PhSH $\left(1.2 \times 10^{-1} \mathrm{~mol} \mathrm{dm}^{-3}\right)$.

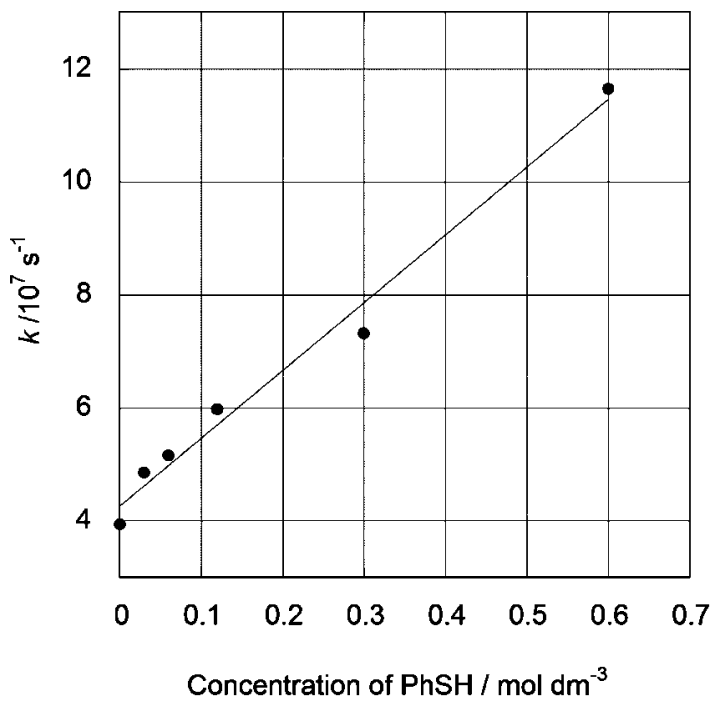

Figure 2. $\mathrm{PhSH}$ concentration dependence of the decay rate $(k)$ observed for the $\mathrm{T}-\mathrm{T}$ absorption of $\mathrm{BP}$ at $525 \mathrm{~nm}$ in a $1 / 9$ mixture of $i$ - $\mathrm{BuOH}$ and $c$ - $\mathrm{HexOH}(\eta=37.3 \mathrm{cP})$ containing $\mathrm{BP}\left(2.0 \times 10^{-2} \mathrm{~mol}\right.$ $\left.\mathrm{dm}^{-3}\right)$ and $\mathrm{PhSH}\left(0.03-0.6 \mathrm{~mol} \mathrm{\textrm {dm } ^ { - 3 }}\right)$.

linear relationship was observed between $k$ and $\mathrm{PhSH}$ concentration. From the slope of this plots, the rate constant for the hydrogen abstraction reaction of ${ }^{3} \mathrm{BP} *$ with $\mathrm{PhSH}$ was determined to be $1.2 \times 10^{8} \mathrm{~s}^{-1} \mathrm{~mol}^{-1} \mathrm{dm}^{3}$, which is close to the rate constant calculated for the diffusion-controlled chemical reaction $\left(k_{\text {dif }}=2 \times 10^{8} \mathrm{~s}^{-1} \mathrm{~mol}^{-1} \mathrm{dm}^{3}\right)$ with $\eta=37.3 \mathrm{cP}^{21}$ Following the disappearance of the $\mathrm{T}-\mathrm{T}$ absorption, the transient absorption attributed to $\mathrm{BPH} \bullet$ was observed at 380 and $550 \mathrm{~nm},{ }^{22}$ and absorption characteristic of $\mathrm{PhS} \bullet$ was also observed at $450 \mathrm{~nm} .{ }^{23}$ Similar results were observed for the other solvents used in the present study. These results indicate that the hydrogen abstraction reaction of ${ }^{3} \mathrm{BP}^{*}$ with $\mathrm{PhSH}$ occurred effectively even in highly viscous solvents.

Magnetic Field Effects. The MFEs on the yield of escaped $\mathrm{BPH} \cdot$ were measured in each solvent under magnetic fields of $0-1.6$ T. Typically, Figure 3 shows the $A(t)$ curves observed at $525 \mathrm{~nm}$ in the absence and presence of a magnetic field of $1.6 \mathrm{~T}$ in (a) $\mathrm{EtOH}(\eta=1.08 \mathrm{cP})$, (b) $i-\mathrm{BuOH}(\eta=3.33 \mathrm{cP})$, and mixtures of $i-\mathrm{BuOH}$ and $c-\mathrm{HexOH}$ at ratios of (c) $1 / 2(\eta=$
$16.9 \mathrm{cP})$ and $(\mathrm{d}) 1 / 50(\eta=55.3 \mathrm{cP})$. Each $A(t)$ curve has two decay components. The first component $(0<t<0.05 \mu \mathrm{s})$ is ascribable to the decay of the $\mathrm{T}-\mathrm{T}$ absorption $\left({ }^{3} \mathrm{BP} *\right)$, and the second component $(0.05 \mu \mathrm{s} \leq t)$ is ascribable to the decay of RPs and escaped $\mathrm{BPH} \cdot$. As shown in Figure $3 \mathrm{a}-\mathrm{c}$, the second decay component was clearly affected by the presence of the magnetic field. As reported previously, these observed MFEs are caused by the $\Delta g \mathrm{M}$ due to the large $\Delta g$ value (0.0052) between $\mathrm{BPH} \bullet(g=2.0030)$ and $\bullet \mathrm{SPh}(g=2.0082)$ and the fast recombination of these RPs. ${ }^{14 a}$ In each solvent, MFEs were generated within $100 \mathrm{~ns}$ after photoexcitation of BP. After the delay time of $100 \mathrm{~ns}$, the magnitude of the MFEs remained nearly constant as the RPs and the escaped $\mathrm{BPH} \bullet$ decayed. These results indicate that MFEs were generated in geminate RPs but that free RPs did not induce the MFEs observed in the present photochemical reaction systems.

Since the lifetime of RPs was within $100 \mathrm{~ns}$, the $A(0.75 \mu \mathrm{s})$ value is proportional to the escaped radical yield for a given magnetic field $(Y(B))$. Thus, the relative yield $R(B)=Y(B) / Y(0$ $\mathrm{T})=A(0.75 \mu \mathrm{s}, B \mathrm{~T}) / A(0.75 \mu \mathrm{s}, 0 \mathrm{~T})$ gives the MFE on the yield of the escaped $\mathrm{BPH} \bullet$. In Figure 4, $R(1.6 \mathrm{~T})$ values observed in the various solvents are plotted against $\eta . R(1.6 \mathrm{~T})$ values decreased with increasing $\eta$ in the range of $0.55 \mathrm{cP} \leq \eta \leq 5$ $\mathrm{cP}$, and then increased with increasing $\eta$ in the range of $5 \mathrm{cp}<$ $\eta \leq 55.3 \mathrm{cP}$. When $\eta$ was higher than $55.3 \mathrm{cP}$, the $R(1.6 \mathrm{~T})$ value became 1.0 , and the MFEs caused by the $\Delta g \mathrm{M}$ were completely quenched. This figure clearly shows that the MFEs caused by the $\Delta g \mathrm{M}$ observed in the present reaction were highly dependent on $\eta$. However, the observed MFEs cannot be explained by the simple diffusion model ${ }^{14}$ because the diffusion model predicts the decrease of $R(1.6 \mathrm{~T})$ with increasing $\eta$ and the saturation of $R(1.6 \mathrm{~T})$ in highly viscous solvents.

Figure 5 depicts the magnetic field dependence of $R(B)$ observed in (a) $\mathrm{EtOH}(\eta=1.08 \mathrm{cP})$, (b) $i-\mathrm{BuOH}(\eta=3.33$ cP), and mixtures of $i-\mathrm{BuOH}$ and $c-\mathrm{HexOH}$ at ratios of (c) $1 / 2$ $(\eta=16.9 \mathrm{cP})$ and $(\mathrm{d}) 1 / 9(\eta=37.3 \mathrm{cP})$. In solvents of low viscosity (Figure $5 \mathrm{a}-\mathrm{c}$ ), $R(B)$ continually decreased with increasing $B$. However, in the highly viscous solvent (Figure $5 \mathrm{~d}$ ), the magnetic field dependence of $R(B)$ exhibited saturation. Specifically, in the $1 / 9$ mixture $(\eta=37.3 \mathrm{cP})$, the MFEs were saturated at $0.6 \mathrm{~T}<B \leq 1.6 \mathrm{~T}$. Similar saturation of the MFEs caused by the $\triangle g \mathrm{M}$ has been reported for the reaction of MBP with $\mathrm{PhSH}$ in $i-\mathrm{BuOH}(\eta=3.33 \mathrm{cP})$ under magnetic fields of greater than $20 \mathrm{~T}$, and this saturation was explained by the simple diffusion model. ${ }^{14}$ In the previous analysis, the saturation field was highly dependent on $\eta$ and decreased with increasing $\eta$. Therefore, the saturation of the MFEs observed in the present study can be qualitatively explained by the diffusion model in the same manner. However, the $R(B)$ value at the saturation field is far from that predicted by the diffusion model $(R(B)=$ 0.66 at saturation).

Analysis of MFEs with the Stochastic Liouville Equation. The anomalous dependence of MFEs on $\eta$ (Figure 4) can be explained by strong spin-orbit coupling (SOC). In the present photochemical reaction of $\bullet \mathrm{SPh}$, the localization of the unpaired spin on the heavy sulfur atom induces strong SOC. ${ }^{24}$ In addition to the change in the isotropic $g$ value of $\bullet \mathrm{SPh}$, this strong SOC causes the following spin dynamics in the RPs: (1) acceleration of spin relaxation by increasing anisotropy of the $g$ tensor; (2) acceleration of spin relaxation by enhancing spin rotational coupling; and (3) mediation of the recombination of triplet RPs. ${ }^{25-27}$ These three effects have been known to quench the MFEs for RPs containing heavy atoms. ${ }^{25}$ 

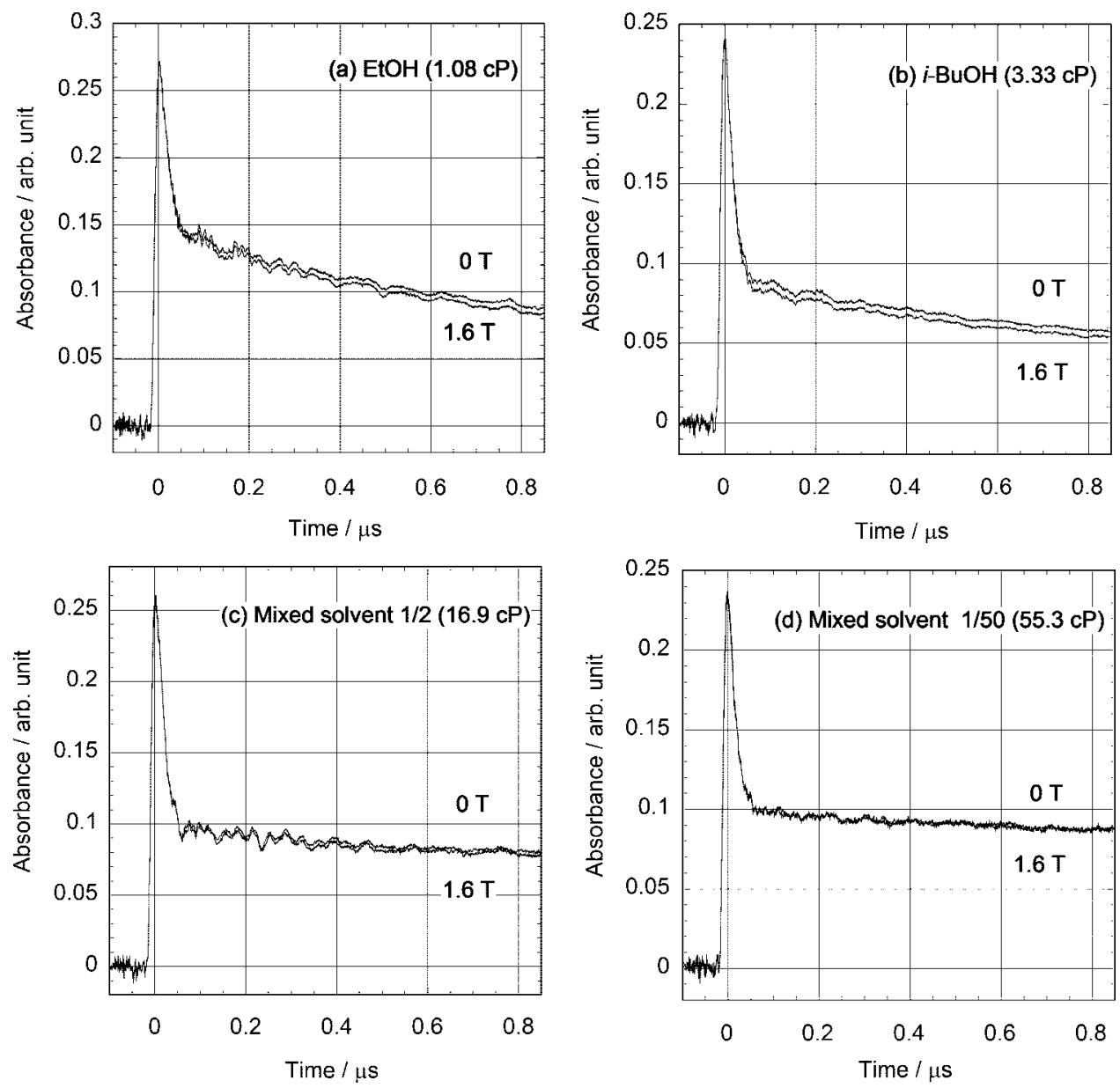

Figure 3. $A(t)$ curves observed at $525 \mathrm{~nm}$ in the absence and presence of a magnetic field of $1.6 \mathrm{~T}$ in (a) $\mathrm{EtOH}(\eta=1.08 \mathrm{cP}),(\mathrm{b}) i-\mathrm{BuOH}(\eta=$ $3.33 \mathrm{cP})$, and mixtures of $i-\mathrm{BuOH}$ and $c-\mathrm{HexOH}$ at ratios of (c) $1 / 2(\eta=16.9 \mathrm{cP})$ and $(\mathrm{d}) 1 / 50(\eta=55.3 \mathrm{cP})$.

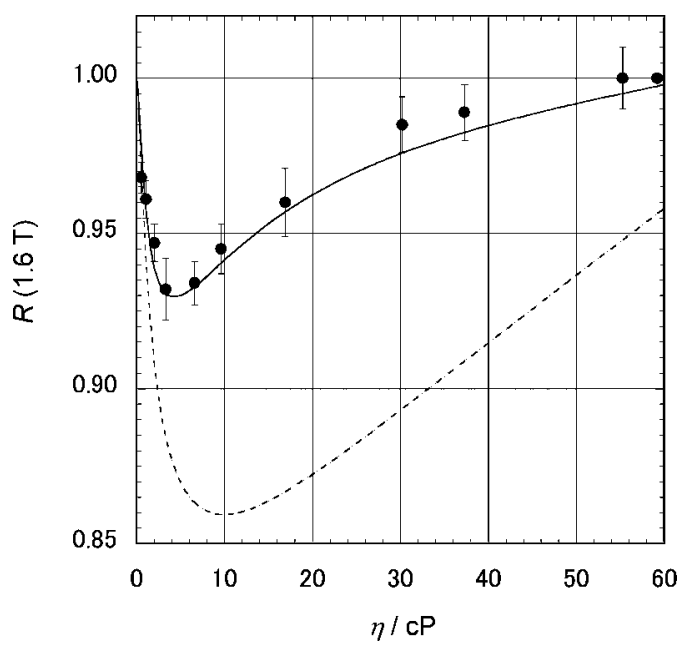

Figure 4. Relationship between $R(1.6 \mathrm{~T})(=Y(1.6 \mathrm{~T}) / Y(0 \mathrm{~T})=A(0.75$ $\mu \mathrm{s}, 1.6 \mathrm{~T}) / A(0.75 \mu \mathrm{s}, 0 \mathrm{~T}))$, observed at $525 \mathrm{~nm}$ for escaped $\mathrm{BPH} \bullet$, and solvent viscosity $(\eta)$. Solid and dashed lines show simulated curves in the presence and absence, respectively, of spin-orbit-couplingmediated recombination of triplet radical pairs $\left(k_{\mathrm{SOC}}=4 \times 10^{9} \mathrm{~s}^{-1}\right)$, with parameters of $J_{0}=1 \times 10^{12} \mathrm{rad} \mathrm{s}^{-1}, \gamma=3, A=0.4 \mathrm{mT}$, and $\delta A$ $=0.2 \mathrm{mT}$.

To clarify the origin of the observed MFEs quenching in highly viscous solvents, we numerically simulated the $\eta$ dependence of the MFEs with the SLE. ${ }^{28-32}$ The model employed in SLE analysis is shown schematically in Scheme 1. Through the hydrogen abstraction reaction, the closely spaced RPs with radicals in contact with each other (denoted "close
RPs" in Scheme 1) are produced with triplet spin multiplicity. In these close RPs, the $\mathrm{S}$ and $\mathrm{T}$ states cannot be mixed because the exchange interaction between them is of high energy. Radicals diffuse from the close RPs, producing separated RPs. In the separated RPs, the $\mathrm{S}$ and $\mathrm{T}$ states are nearly degenerated, and the $\mathrm{S}-\mathrm{T}$ mixing is promoted by the $\Delta g \mathrm{M}$, the hyperfine coupling mechanism (HFCM), and spin relaxations. By means of diffusional motion, the separated RPs either escape to form escaped radicals or reform close RPs. Recombination reactions are assumed to involve close RPs. In the absence of the SOC, only the singlet close RPs recombine with an associated rate constant of $k_{\text {rec }}$ (eq 4). In the presence of the SOC, the triplet close RPs can also recombine with an associated rate constant of $k_{\text {SOC }}$.

$$
{ }^{3}(\mathrm{BPH} \bullet \bullet \mathrm{SPh}) \stackrel{k_{\mathrm{SOC}}}{\longrightarrow} \text { recombination products }
$$

MFEs on the chemical reactions generally occur from the following steps: (1) the magnetic field affects the T-S spin conversion in the separated RPs, and (2) the subsequent spinstate selective recombination from the singlet state influences the extent of MFE on the yield of escaped radicals. However, in the case of SOC-mediated recombination (eq 7), there is no spin selectivity of recombination, and thus the MFEs are quenched.

The details of the SLE analysis are described in Supporting Information. In the present study, we used the Pedersen-Freed type SLE, ${ }^{28}$ in which the effects of spin-spin interactions, 

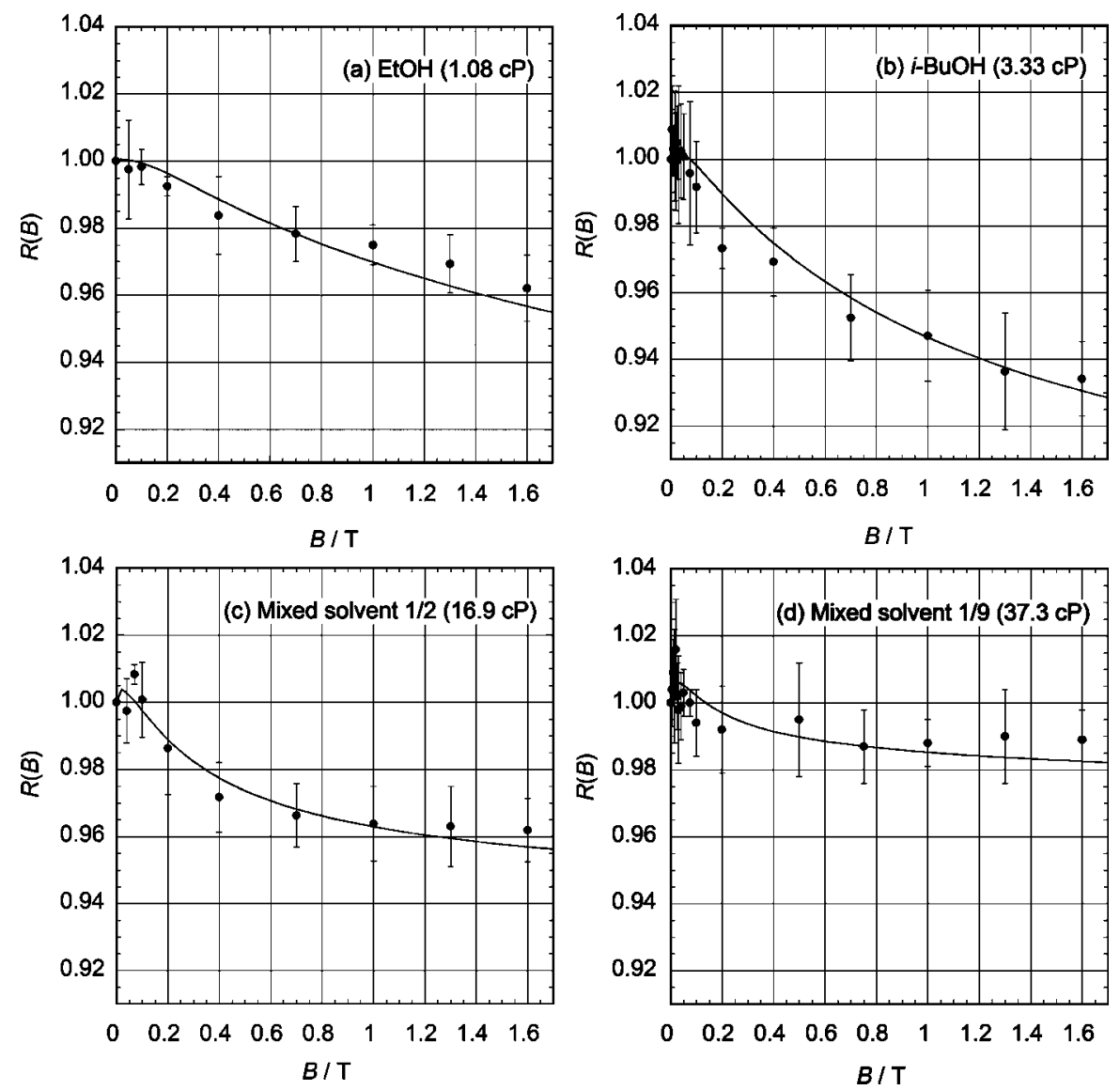

Figure 5. Magnetic field dependence of $R(B)$ observed at $525 \mathrm{~nm}$ for escaped $\mathrm{BPH} \bullet$ in (a) EtOH $(\eta=1.08 \mathrm{cP})$, (b) $i$-BuOH $(\eta=3.33 \mathrm{cP})$, and mixture of $i-\mathrm{BuOH}$ and $c-\mathrm{HexOH}$ at ratios of $(\mathrm{c}) 1 / 2(\eta=16.9 \mathrm{cP})$ and $(\mathrm{d}) 1 / 9(\eta=37.3 \mathrm{cP})$. Solid lines show simulated curves obtained from the SLE analysis.

molecular diffusion, recombination reactions, and spin relaxations were included as follows:

$$
\frac{\partial \rho(r, t)}{\partial t}=-i H(r)^{\times} \rho(r, t)+D \Gamma_{r} \rho(r, t)+K_{r} \rho(r, t)+R_{r} \rho(r, t)
$$

In this equation, $H(r)^{\times}$is the commutator associated with the spin Hamiltonian $H(r)$ at distance $r . \rho(r, t)$ is density matrix of the RP at time $t$ and distance $r . D$ and $\Gamma_{r}$ are the diffusion coefficient and the diffusion operator, respectively. $K_{r}$ and $R_{r}$ are the superoperators for the recombination reactions and the spin relaxations, respectively.

The Laplace transform of eq 8 is

$$
\begin{array}{r}
s \hat{\rho}(r, s)-\rho(r, t=0)=-i H(r)^{\times} \hat{\rho}(r, s)+D \frac{\partial^{2}}{\partial r^{2}} \hat{\rho}(r, s)+ \\
K \hat{\rho}(r, s)+R \hat{\rho}(r, s)
\end{array}
$$

where

$$
\hat{\rho}(r, s) \equiv \int_{0}^{\infty} e^{-s t} r \rho(r, t) d t \equiv \int_{0}^{\infty} e^{-s t} \hat{\rho}(r, t) d t
$$

Since the magnitudes of the MFEs were nearly constant after a delay time of $100 \mathrm{~ns}$, the experimental data used for SLE simulations were obtained at a delay time of $0.75 \mu \mathrm{s}$. Therefore, only the limiting condition of $t \rightarrow \infty$ was used. The yield of escaped radicals $\left(Y_{\text {calc }}\right)$ is given by

$$
Y_{\mathrm{calc}}=\lim _{s \rightarrow 0} s \operatorname{Tr}\left[\int_{d}^{\infty} r \hat{\rho}(r, s) d r\right]
$$

The MFEs on the yield of escaped radicals $\left(R_{\text {calc }}\right)$ are then calculated as
SCHEME 1: Schematic Representation of the Model Used for SLE Analysis

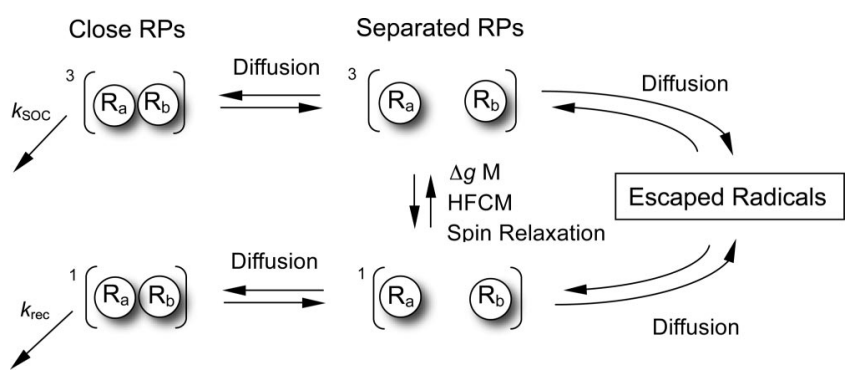

$$
R_{\text {calc }}(B)=\frac{Y_{\text {calc }}(B \mathrm{~T})}{Y_{\text {calc }}(0 \mathrm{~T})}
$$

The spin Hamiltonian used here is composed of the Zeeman interactions for the radicals, the hyperfine interactions between electron and nuclear spins with a HFC constant $(A)$, and the $r$-dependent exchange interaction $(J)$ :

$$
H(r)=\mu_{\mathrm{B}} \hbar^{-1} B\left(g_{\mathrm{a}} S_{\mathrm{az}}+g_{\mathrm{b}} S_{\mathrm{bz}}\right)+A \mathbf{S}_{\mathrm{a}} \mathbf{I}_{\mathrm{a}}-J(r)\left(\frac{1}{2}+2 \mathbf{S}_{\mathrm{a}} \mathbf{S}_{\mathrm{b}}\right)
$$

Here, $\mathbf{S}$ and $\mathbf{I}$ represent the electron and the nuclear spin operators, respectively. Subscripts a and b denote the individual radicals (a for $\mathrm{BPH} \bullet$ and $\mathrm{b}$ for $\mathrm{PhS} \bullet$ ). $g_{\mathrm{a}}$ and $g_{\mathrm{b}}$ are the isotropic $g$ factors for radical a and b, respectively. In the present reaction, the MFEs due to the HFCM have hardly been observed. ${ }^{14,33}$ For simplicity, one magnetic nucleus in radical a was included, 
and the HFC constant was used for the fitting parameter. The $r$ dependence of $J$ is treated with the exponential function as:

$$
J(r)=J_{0} \exp [-\beta(r-d)]
$$

Here, $J_{0}$ denotes the magnitude of the exchange interaction at the closest distance $d$.

The diffusion of the radicals in RPs was assumed to proceed by simple Brownian motion and was treated by the finite difference technique with mutual diffusion coefficient $D$, which is the sum of the diffusion coefficients for radicals a and b $(D$ $\left.=D_{\mathrm{a}}+D_{\mathrm{b}}\right)$. For studies of radical diffusion by the TG technique, Terazima et al. reported that the diffusion of radicals is described by the Stokes-Einstein equation (eq 15), ${ }^{9}$ whereas the $\eta$ and solute-size dependences of stable molecule diffusion are described by the empirical equation proposed by Evans et al. ${ }^{34}$ For the SLE analysis, we used the Stokes-Einstein equation to calculate the $\eta$ dependence of the diffusion coefficient for radicals. The diffusion coefficient for each radical is represented by the Stokes-Einstein equation as follows:

$$
D_{\mathrm{a}}(\eta)=\frac{k_{\mathrm{B}} T}{6 \pi \eta d_{\mathrm{a}}}, \quad D_{\mathrm{b}}(\eta)=\frac{k_{\mathrm{B}} T}{6 \pi \eta d_{\mathrm{b}}}
$$

Here, $d_{\mathrm{a}}$ and $d_{\mathrm{b}}$ are the radii of the respective radicals. The diffusion coefficient for $\mathrm{BPH} \bullet$ in 2-propanol $(\eta=3.3 \mathrm{cP})$ was observed to be $6.8 \times 10^{-10} \mathrm{~m}^{2} \mathrm{~s}^{-1}$ with the TG technique. ${ }^{9}$ This value corresponds to an $0.4 \mathrm{~nm}$ effective radical radius for diffusion according to eq 15. The diffusion coefficient for $\mathrm{PhS} \bullet$ has not been reported, but we estimated the radius of $\mathrm{PhS} \bullet$ to be $0.2 \mathrm{~nm}$ using the diffusion coefficient reported for a benzyl radical in cyclohexane. ${ }^{11}$ In the SLE analysis, however, we could not reproduce the experimental results with $d_{\mathrm{a}}=0.4 \mathrm{~nm}$ and $d_{\mathrm{b}}=0.2 \mathrm{~nm}$. The major discrepancy was that the absolute magnitude of the MFEs obtained from the SLE simulation with the parameters of $d_{\mathrm{a}}=0.4 \mathrm{~nm}$ and $d_{\mathrm{b}}=0.2 \mathrm{~nm}$ was smaller than that experimentally observed. To reproduce the experimental results, we introduced a correlation factor $(\gamma)$ for the radical radius as follows,

$$
d_{\mathrm{a}}=\gamma a_{\mathrm{a}}, \quad d_{\mathrm{b}}=\gamma a_{\mathrm{b}}
$$

Here $a_{\mathrm{a}}$ and $a_{\mathrm{b}}$ are the radical radii expected from the TG technique $\left(a_{\mathrm{a}}=0.4 \mathrm{~nm}\right.$ and $\left.a_{\mathrm{b}}=0.2 \mathrm{~nm}\right) . d_{\mathrm{a}}$ and $d_{\mathrm{b}}$ are radical radii that produce diffusion coefficients that are effective for the generation of the MFEs with the assumption of simple Brownian motion. The $K_{r}$ matrix includes recombination reactions from the singlet RPs with the rate constant $k_{\text {rec }}$ and those from the triplet RPs with the rate constant $k_{\mathrm{SOC}}$ at the closest distance $d$. In the $R_{r}$ matrix, the spin relaxations by anisotropy $(\delta A)$ of the hyperfine interaction, the anisotropies $(\delta g)$ of the $g$ factors, and the spin rotational interactions were taken into account. We assumed a $\delta A$ value of one-half of the $A$ value.

For the simulation, reported values of $g_{\mathrm{a}}=2.003, g_{\mathrm{b}}=2.0082$ were used for the magnetic parameters of $\mathrm{BPH} \bullet$ and $\mathrm{PhS} \bullet$, respectively. ${ }^{3,35}$ The several parameter sets have been reported for the $r$-dependence of the $J$ values in RPs. ${ }^{26,32,36-38}$ The typical parameters for the $J$ value used in the SLE analysis are $J_{0}=10^{10}-10^{11} \mathrm{rad} \mathrm{s}^{-1}$ and $\beta=20 \mathrm{~nm}^{-1}$. $32 \mathrm{c}$ We have used the value of $\beta=20 \mathrm{~nm}^{-1}$ while the $J_{0}$ value was treated as a fitting parameter in the analysis. The spin relaxation rate constants are calculated with the general formulas (see Supporting Information) using the reported values of $\delta g_{\mathrm{a}}=0.002^{39}$ and $\delta g_{\mathrm{b}}=$ $0.02^{19,40}$ except the spin rotational relaxation. The rate constants of the spin rotational relaxations are known to be on the order of $10^{4}-10^{6} \mathrm{~s}^{-1}$ for radicals involving heavy atoms. ${ }^{25} \mathrm{We}$ assumed the spin rotational relaxation is independent of $B$ and $\eta$. The constant value of $2 \times 10^{6} \mathrm{~s}^{-1}$ was used for the relaxation rate constant due to the spin rotational interaction.

The experimental results were fitted with the following parameters: $k_{\mathrm{rec}}, k_{\mathrm{SOC}}, \gamma, J_{0}$, and the HFC values $(A$ and $\delta A) . B$ and $\eta$ dependences of the $R_{\text {calc }}$ values (solid lines) simulated by the SLE with parameters of $k_{\mathrm{rec}}=5 \times 10^{10} \mathrm{~s}^{-141}, k_{\mathrm{SOC}}=4$ $\times 10^{9} \mathrm{~s}^{-141}, J_{0}=1 \times 10^{12} \mathrm{rad} \mathrm{s}^{-1}, \beta=20 \mathrm{~nm}^{-1}, \gamma=3, A=$ $0.4 \mathrm{mT}$, and $\delta A=0.2 \mathrm{mT}$ are shown in Figures 4 and 5 . In the present SLE analysis, we used the $k_{\text {rec }}$ value of $5 \times 10^{10} \mathrm{~s}^{-1}$. The increase of $k_{\text {rec }}$ from $5 \times 10^{10} \mathrm{~s}^{-1}$ had little effect on the simulated results. This $k_{\text {rec }}$ value used for the simulation is close to the rate of the diffusion-controlled reactions, and it is consistent with the previous report. ${ }^{14}$ The $J_{0}$ value of $1 \times 10^{12}$ $\mathrm{rad} \mathrm{s}^{-1}$ was somewhat larger than the typical value of $10^{10}-10^{11}$ rad s ${ }^{-1}$. With $J_{0}=10^{10}-10^{11} \mathrm{rad} \mathrm{s}^{-1}$, we could not reproduced the saturation of the MFEs observed in the highly viscous solvents. The localization of the unpaired spin in $\mathrm{PhS} \bullet$ may cause the relatively large $J_{0}$ value in the present study. The comparable $J_{0}$ values $\left(10^{12}-10^{13} \mathrm{rad} \mathrm{s}^{-1}\right)$ have been reported with detailed SLE analysis on the chemically induced dynamic electron polarization generated in neutral RPs. ${ }^{36}$

The MFEs observed experimentally in the studied solvents were well-reproduced by the SLE analysis, as was the $\eta$ dependence of these MFEs at $1.6 \mathrm{~T}$. In Figure 4, the $\eta$ dependence of the MFEs simulated without SOC-mediated recombination from triplet RPs is also shown (dashed line). The SLE simulations indicate that recombination from the triplet RPs is the main cause of the MFE quenching observed in highly viscous solvents. For close RPs, the recombination reaction competes with the diffusional motion of close RPs to form separated RPs. In solvents of low viscosity, singlet RPs were the main species that proceeded to recombine, because their close RPs had a short lifetime and because $k_{\text {rec }}>k_{\text {SOC }}$. As $\eta$ increased, the lifetime of the close RPs increased, providing enough time to allow recombination from triplet RPs. Therefore, both singlet and triplet RPs recombined in highly viscous solutions, leading to quenching of the MFEs.

The magnitude of the $k_{\text {SOC }}$ obtained from the SLE analysis was somewhat large. Since the value of $k_{\mathrm{rec}}$ and $k_{\mathrm{SOC}}$ depend on segment size for $r$ at contact in SLE, only relative magnitudes of $k_{\text {rec }}$ and $k_{\mathrm{SOC}}$ are physically meaningful. ${ }^{26,38 \mathrm{a}}$ In the present study $\bullet \mathrm{SPh}$ radicals contain heavy sulfur atom and the unpaired spin is localized on the sulfur atom. ${ }^{24}$ Therefore, $k_{\text {SOC }}$ should become larger than those of ordinary $\mathrm{C}$-centered radicals. The SOC mediated recombination reaction from the triplet RPs has been observed with a RP system involving $\mathrm{Cl}$ atom even in acetonitrile solvents $(\eta<0.5 \mathrm{cP})$ where the lifetime of the contact RP is much shorter than those in the alcoholic solvents used in the present study. ${ }^{42}$ Therefore, the obtained $k_{\mathrm{SOC}}$ value seems to be reasonable for the RPs containing the sulfur atom. Moreover, the $k_{\mathrm{SOC}}$ value is much higher than the triplet decay rate of $\mathrm{BP}$ in the absence of PhSH $\left(4 \times 10^{7} \mathrm{~s}^{-1}\right)$. Since the present hydrogen abstraction reaction of $\mathrm{BP}$ with $\mathrm{PhSH}$ is the diffusion controlled reaction, ${ }^{14}$ the hydrogen abstraction reaction ${ }^{3} \mathrm{BP}^{*}$ can compete with such fast SOC-induced quenching of ${ }^{3} \mathrm{BP} *$.

Figure 4 shows that the MFEs observed in the highly viscous range of $\eta>10 \mathrm{cP}$ are quenched even when the SOC-mediated recombination from triplet RPs was not included in the simulation (dashed line). This result indicates that the spin relaxations by $\delta A$ and $\delta g$ and the spin rotational relaxation also contribute to quenching of the MFEs. However, the rates of spin relaxation by $\delta A$ and of the spin rotational relaxation are 
unknown. Using SLE analysis, we examined how those relaxations affected the MFEs. ${ }^{43}$

First, to clarify the effect of spin relaxation by $\delta A$, we tried to fit the experimental results by adjusting the value of $\delta A$ without SOC-mediated recombination from the triplet RPs. However, we could not reproduce the $B$ dependence of the MFEs $(R(B))$ observed in the various solvents. Therefore, we excluded the possibility of MFE quenching by spin relaxation induced by $\delta A$. Next, the effect of the spin rotational relaxation on MFEs was examined. We have also tried to fit the experimental data with increasing the rate constant for the spin rotational relaxation. We could reproduce the $\eta$ dependence of the MFEs at $1.6 \mathrm{~T}$; however, we could not reproduce the $B$ dependence of the MFEs $(R(B))$ observed in the various solvents; the saturation of the MFEs could not be reproduced in the highly viscous solvents. Therefore, spin rotational relaxation is not the cause of the quenching of MFEs observed in the present study.

The good agreement between the experimental data and the results of SLE analysis shows that the $\eta$ dependence of radical diffusion was well-described by the Stokes-Einstein equation with $\gamma=3$. From the TG measurements, Terazima et al. reported that radical diffusion is described by the Stokes-Einstein equation with a value of $\gamma=1$. In the TG technique, the observed radical diffusion is averaged over a time period longer than microseconds. Therefore, radical diffusion coefficients obtained in this manner are associated with macroscopic solvent viscosity. In contrast, MFEs are affected by the microviscosity in the vicinity of RPs, because the spin-spin interactions in RPs are limited to nanoscale distances. The parameter of $\gamma=3$ used for the SLE simulation performed here therefore suggests that the microviscosity in the vicinity of the radical pairs should be larger than the solvent's macroscopic viscosity. Theoretical analyses have predicted that the hydrodynamic effects, the effects of the solvent structures, or both slow the relative diffusion of solutes at short solute-solute distances in molecular liquids. ${ }^{44-46}$ More recently, Tavernier et al. investigated photoinduced bimolecular electron transfer reactions by fluorescence spectroscopy. ${ }^{47}$ In this report as well, solute diffusion was found to be restricted by the hydrodynamic effects and the effects of the solvent structures at short solute-solute distances. Thus, we concluded that slow radical diffusion, which is obtained with the assumption of simple Brownian motion in the present study, is attributed to the hydrodynamic effects, the effects of the solvent structures at short radical-radical distances, or both. Furthermore, the SLE analysis showed that the MFEs caused by the $\triangle g \mathrm{M}$ and the quenching of the MFEs by the SOC reflect radical diffusion over short distances.

\section{Conclusion}

The $\eta$ dependence of MFEs caused by the $\Delta g \mathrm{M}$ on the reaction of triplet $\mathrm{BP}$ with $\mathrm{PhSH}$ was studied by nanosecond laser flash photolysis and SLE analysis. The observed MFEs were highly sensitive to $\eta$. SLE analysis revealed that the MFEs were quenched by SOC-mediated recombination of triplet RPs in highly viscous solvents. The radical diffusion associated with the MFEs was interpreted with the Stokes-Einstein equation with the modification of the radical radii. The diffusion coefficients for the radicals estimated from the SLE analysis were about three times smaller than those estimated from the macroscopic viscosity, indicating that the microviscosity in the vicinity of the RPs should be larger than the solvent's macroscopic viscosity. The present study demonstrates that the microviscosity in confined and inhomogeneous systems can be probed through the observation of MFEs on the present photochemical reaction.

Acknowledgment. This work was partially supported by a Grant-in-Aid for Scientific Research (No. 20031005) in Priority Area "Science of Ionic Liquids" (Area No. 452) from the Ministry of Education, Culture, Sports, Science and Technology of Japan. T.Y. acknowledges partial support from a Grant-inAid for Young Scientists (Start-up, No. 19850004) from the Ministry of Education, Culture, Sports, Science and Technology of Japan.

Supporting Information Available: Details of the SLE analysis. This material is available free of charge via the Internet at http://pubs.acs.org.

\section{References and Notes}

(1) Steiner, U. E.; Ulrich, T. Chem. Rev. 1989, 89, 51-147.

(2) Nagakura, S.; Hayashi, H.; Azumi, T. Dynamic Spin Chemistry; Kodansha-Wiley: Tokyo, NY, 1998.

(3) Hayashi, H. Introduction to Dynamic Spin Chemistry; World Scientific: Singapore, 2004.

(4) Kitahama, Y.; Wakasa, M.; Sakaguchi, Y. J. Phys. Chem. A 2004, $108,754-757$.

(5) Gohdo, M.; Kitahama, Y.; Sakaguchi, Y.; Wakasa, M. J. Photochem. Photobiol. A: Chem. 2008, 199, 130-135.

(6) Okazaki, M.; Toriyama, K.; Oda, K.; Kasai, T. Phys. Chem. Chem. Phys. 2002, 4, 1201-1205.

(7) (a) Wakasa, M. J. Phys. Chem. B 2007, 111, 9434-9436. (b) Hamasaki, A.; Yago, T.; Takamasu, T.; Kido, G.; Wakasa, M. J. Phys. Chem. B 2008, 112, 3375-3379.

(8) Cussler, E. L. Diffusion: Mass transfer in fluid systems; Cambridge University Press: Cambridge, 1997.

(9) (a) Terazima, M.; Okamoto, K.; Hirota, N. J. Phys. Chem. 1993, 97, 13387-13393. (b) Terazima, M. Acc. Chem. Res. 2000, 33, 687-694. (c) Okamoto, K.; Hirota, N.; Tominaga, T.; Terazima, M. J. Phys. Chem. A 2001, 105, 6586-6593.

(10) (a) Noyes, R. M. J. Am. Chem. Soc. 1959, 81, 566-570. (b) Levison, S. A.; Noyes, R. M. J. Am. Chem. Soc. 1964, 86, 4525-4529. (c) Noyes, R. M. J. Phys. Chem. 1965, 69, 3182-3183.

(11) (a) Burkhart, R. D.; Boynton, R. F.; Merrill, J. C. J. Am. Chem. Soc. 1971, 93, 5013-5017. (b) Burkhart, R. D.; Wong, R. J. J. Am. Chem. Soc. 1973, 95, 7203-7206.

(12) Galtseva, E. V.; Yakimchenko, O. Y.; Lebedev, Y. S. Chem. Phys. Lett. 1983, 99, 301-304.

(13) Hornak, J. P.; Moscicki, J. K.; Schneider, D. J.; Freed, J. H. J. Chem. Phys. 1986, 84, 3387-3395.

(14) (a) Wakasa, M.; Hayashi, H. J. Phys. Chem. 1996, 100, 1564015643. (b) Wakasa, M.; Nishizawa, K.; Abe, H.; Kido, G.; Hayashi, H. J. Am. Chem. Soc. 1998, 120, 10565-10566. (c) Wakasa, M.; Nishizawa, K.; Abe, H.; Kido, G.; Hayashi, H. J. Am. Chem. Soc. 1999, 121, 91919197.

(15) (a) Wolff, H.-J.; Bür $\beta$ ner, D.; Steiner, U. E. Pure Appl. Chem. 1995, 67, 167-174. (b) Krissinel, E. B.; Burshtein, A. I.; Lukzen, N. N.; Steiner, U. E. Mol. Phys. 1999, 96, 1083-1097.

(16) Freed, J. H. Chemically Induced Magnetic Polarization; Muss, L. T.; Atkins P. W.; McLauchlan, K. A.; Pedersen, J. B., Eds.; Reidel: Dorderecht, 1977; Chapter 19.

(17) Pedersen, J. B. J. Chem. Phys. 1977, 67, 4097-4102.

(18) Riddick, J. A.; Bunger, W. B.; Sakano, T. K. Organic Solvents, 4th ed.; Wiley: New York, 1986.

(19) Hamasaki, A.; Sakaguchi, Y.; Nishizawa, K.; Kido, G.; Wakasa, M. Mol. Phys. 2006, 104, 1765-1771.

(20) Carmichael, I.; Hug, G. L. J. Phys. Chem. Ref. Data 1968, 15, $1-250$.

(21) The diffusion controlled reaction rate $\left(k_{\mathrm{dif}}\right)$ was calculated to be 2 $\times 10^{8} \mathrm{~s}^{-1} \mathrm{~mol}^{-1} \mathrm{dm}^{3}$ by the relation of $k_{\text {dif }}=4 \pi d \times\left(k_{\mathrm{B}} T / 6 \pi \eta d\right)$ with the parameters of $d=0.6 \mathrm{~nm}, T=296 \mathrm{~K}$, and $\eta=37.3 \mathrm{cP}$.

(22) Sakaguchi, Y.; Hayashi, H.; Nagakura, S. J. Phys. Chem. 1982, $86,3177-3184$.

(23) Jeschke, G.; Wakasa, M.; Sakaguchi, Y.; Hayashi, H. J. Chem. Phys. 1994, 98, 4069-4075.

(24) (a) Lim, J. S.; Lim, I. S.; Lee, K.-S.; Ahn, D.-S.; Lee, Y.-S.; Kim, S. K. Angew. Chem., Int. Ed. 2006, 45, 6290-6293. (b) Lim, I. S.; Lim, J. S.; Lee, Y. S.; Kim, S. K. J. Chem. Phys. 2007, 126, 034306.

(25) Khudyakov, I. V.; Serebrennikov, Y. A.; Turro, N. J. Chem. Rev. 1993, 93, 537-570. 
(26) de Kanter, F. J. J.; Kaptein, R. J. Am. Chem. Soc. 1982, 104, 47594766 .

(27) Cross, G. L.; Redwine, O. D. J. Am. Chem. Soc. 1985, 107, 61316133.

(28) (a) Pedersen, J. B.; Freed, J. H. J. Chem. Phys. 1973, 58, 27462762. (b) Pedersen, J. B.; Freed, J. H. J. Chem. Phys. 1973, 59, 28692885 .

(29) Busmann, H.-G.; Staerk, H.; Weller, A. J. Chem. Phys. 1989, 91, 4098-4105.

(30) (a) Kobori, Y.; Akiyama, K.; Tero-Kuobta, S. J. Chem. Phys. 2000, 113, 465-468. (b) Kobori, Y.; Akiyama, K.; Yago, T.; Tero-Kuobta, S. J. Am. Chem. Soc. 2001, 123, 9722-9723. (c) Yago, T.; Kobori, Y.; Akiyama, K.; Tero-Kubota, S. J. Phys. Chem. B 2002, 106, 10074-10081. (d) Kobori, Y.; Yago, T.; Tero-Kuobta, S. Appl. Magn. Reson. 2003, 23, 269-287. (e) Kobori, Y.; Yago, T.; Tero-Kubota, S.; Sato, H.; Hirata, F.; Norris, J. R. J. Phys. Chem. B 2004, 108, 10226-10240.

(31) (a) Ito, F.; Ikoma, T.; Akiyama, K.; Kobori, Y.; Tero-Kubota, S. J. Am. Chem. Soc. 2003, 125, 4722-4723. (b) Ito, F.; Ikoma, T.; Akiyama, K.; Tero-Kubota, S. J. Phys. Chem. B 2005, 109, 7208-7213. (c) Ito, F Ikoma, T.; Akiyama, K.; Watanabe, A.; Tero-Kubota, S. J. Phys. Chem. B 2005, 109, 8707-8717. (d) Ito, F.; Ikoma, T.; Akiyama, K.; Watanabe, A. Tero-Kubota, S. J. Phys. Chem. B 2006, 110, 5161-5162.

(32) (a) Tarasov, V. F.; Ghatlia, N. D.; Buchachenko, A. L.; Turro, N. J. J. Am. Chem. Soc. 1992, 114, 9517-9526. (b) Tarasov, V. F.; Ghatlia, N. D. Avdievich, N. I.; Shkrob, I. A.; Buchachenko, A. L.; Turro, N. J. J. Am. Chem. Soc. 1994, 116, 2281-2291. (c) Tarasov, V. F.; Bagranskaya, E. G.; Shkrob, I. A.; Avdievich, N. I.; Ghatlia, N. D.; Lukuzen, N. N.; Turro, N. J.; Sagdeev, R. Z. J. Am. Chem. Soc. 1995, 117, 110-118.

(33) Wakasa, M.; Sakaguchi, Y.; Hayashi, H. Chem. Lett. 1994, 23, 4950 .

(34) Chen, S. H.; Davis, H. T.; Evans, D. F. J. Chem. Phys. 1982, 77, $2540-2544$
(35) Mörle, W.; Jezierski, A.; Singer, H. Z. Chem. 1979, 19, 147-148.

(36) Kitahama, Y.; Kimura, Y.; Hirota, N. Bull. Chem. Soc. Jpn. 2000, $73,851-859$

(37) Okazaki, M.; Toriyama, K. J. Phys. Chem. 1995, 99, 17244-17250.

(38) (a) Tsentalovich, Y. P.; Morozova, O. B.; Avdievich, N. I.; Ananchenko, G. S.; Yurkovskaya, A. V.; Ball, J. D.; Forbes, M. D. E J. Phys. Chem. A 1997, 101, 8809-8816. (b) Tsentalovich, Y. P.; Forbes, M. D. E.; Morozova, O. B.; Plotnikov, I. A.; McCaffrey, V. P.; Yurkovskaya, A. V. J. Phys. Chem. A 2002, 106, 7121-7129.

(39) (a) Nakamura, Y.; Igarashi, M.; Sakaguchi, Y.; Hayashi, H. Chem. Phys. Lett. 1994, 217, 387-392. (b) Nishizawa, K.; Sakaguchi, Y.; Abe, H.; Kido, G.; Hayashi, H. Mol. Phys. 2002, 100, 1137-1146.

(40) Wakasa, M.; Hayashi, H.; Mikami, Y.; Takeda, T. J. Phys. Chem. 1995, 99, 13181-13186.

(41) In the SLE analysis, the reactivity of the singlet and triplet RPs were determined by the values of $k_{\text {rec }}$ and $k_{\mathrm{SOC}}, D$, and reaction zone $(\Delta r)$ at the contact. We used $\Delta r=0.03 \mathrm{~nm}$ for the singlet and triplet RPs in the SLE analysis.

(42) Kikuchi, K. Hoshi, M.; Abe, E.; Kokubun, H. J. Photochem. Photobiol. A: Chem. 1988, 45, 1-7.

(43) SLE analysis showed that the MFEs due to the HFCM were not sensitive to $\eta$.

(44) Deutch, J. M.; Felderhof, B. U. J. Chem. Phys. 1973, 59, 16691671

(45) Northrup, S. H.; Hynes, J. T. J. Chem. Phys. 1979, 71, 871-882.

(46) Wolynes, P. G.; Deutch, J. M. J. Chem. Phys. 1976, 65, 450.

(47) (a) Tavernier, H. L.; Kalashnikov, M. M.; Fayer, M. D. J. Chem. Phys. 2000, 113, 10191-10201. (b) Tavernier, H. L.; Fayer, M. D. J. Chem. Phys. 2001, 143, 4552-4564.

JP804862W 